[7] J. Bitzer, K.-D. Kammeyer, and K. U. Simmer, "An alternative implementation of the superdirective beamformer," in Proc. IEEE Workshop Application Signal Process. Audio Acoust., New Paltz, NY, Oct. 1999.

[8] C. Marro, Y. Mahieux, and K. U. Simmer, "Analysis of noise reduction and dereverberation techniques based on microphone arrays with postfiltering," IEEE Trans. Speech Audio Processing, vol. 6, pp. 240-259, May 1998.

[9] S. Nordholm, I. Claeson, and M. Dahl, "Adaptive microphone array employing calibration signals: an analytical evaluation," IEEE Trans. Antennas Propagat., vol. 8, pp. 975-981, Aug. 1992.

[10] K.-C. Huarng and C.-C. Yeh, "Performance analysis of derivative constraint adaptive arrays with pointing errors," IEEE Trans. Antennas Propagat., vol. 40, pp. 975-981, Feb. 1992.

[11] S. Gannot, D. Burshtein, and E. Weinstein, "Theoretical performance analysis of the general transfer function GSC," Technion-Israel Inst. Techno., Haifa, Israel, CCIT Rep. 381, May 2002.

[12] - "Theoretical analysis of the general transfer function GSC," in Proc. Int. Workshop Acoust. Echo Noise Contr., Darmstadt, Germany, Sept. 2001

[13] S. Nordholm, I. Claesson, and P. Eriksson, "The broadband wiener solution for Griffiths-Jim beamformers," IEEE Trans. Signal Proc., vol. 40, pp. 474-478, Feb. 1992.

[14] O. Shalvi and E. Weinstein, "System identification using nonstationary signals," IEEE Trans. Signal Proc., vol. 44, pp. 2055-2063, Aug. 1996.

\section{Least Squares Algorithms for Time-of-Arrival-Based Mobile Location}

\author{
K. W. Cheung, H. C. So, W.-K. Ma, and Y. T. Chan
}

\begin{abstract}
Localization of mobile phones is of considerable interest in wireless communications. In this correspondence, two algorithms are developed for accurate mobile location using the time-of-arrival measurements of the signal from the mobile station received at three or more base stations. The first algorithm is an unconstrained least squares (LS) estimator that has implementation simplicity. The second algorithm solves a nonconvex constrained weighted least squares (CWLS) problem for improving estimation accuracy. It is shown that the CWLS estimator yields better performance than the LS method and achieves both the Cramér-Rao lower bound and the optimal circular error probability at sufficiently high signal-to-noise ratio conditions.
\end{abstract}

Index Terms-Mobile terminals, positioning algorithms, time-of-arrival.

\section{INTRODUCTION}

Mobile location has received significant interest since the first ruling of the Federal Communications Commission for detection of emergency calls in the United States in 1996 [1]. In addition to emergency

Manuscript received September 9, 2002; revised June 24, 2003. This work was supported by a Grant from the Research Grants Council of the Hong Kong Special Administrative Region, China under Project CityU 1119/01E. The associate editor coordinating the review of this manuscript and approving it for publication was Prof. Randolph L. Moses.

K. W. Cheung and H. C. So are with the Department of Computer Engineering and Information Technology, City University of Hong Kong, Kowloon, Hong Kong (e-mail: gary.cheung@student.cityu.edu.hk; ithcso@cityu.edu.hk).

W.-K. Ma is with the Department of Electrical and Electronic Engineering, the University of Melbourne, Parkville, Australia (e-mail: w.ma@ee.mu.oz.au).

Y. T. Chan is with the Department of Electronic Engineering, The Chinese University of Hong Kong, Shatin, N.T., Hong Kong (e-mail: wkma@ee.cuhk.edu.hk; ytchan@ee.cuhk.edu.hk).

Digital Object Identifier 10.1109/TSP.2004.823465 management, mobile position information will also be useful in intelligent transport systems, location billing, interactive map consultation, and monitoring of the mentally impaired [2]-[6].

Wireless location systems usually require two or more base stations (BSs) to intercept a mobile station (MS) signal. Common location approaches are based on time-of-arrival (TOA), received signal strength (RSS), time-difference-of-arrival (TDOA), or angle-of-arrival (AOA) measurements determined from the MS signals received at the BSs [6]-[10]. In this correspondence, we focus on mobile positioning using the TOA information.

In the TOA method, the one-way propagation time of the signal traveling between the MS and each of the BSs is measured, and this provides a circle centered at the BS on which the MS must lie. The TOA measurements are then converted into a set of circular equations, from which the MS position can be determined with the knowledge of the BS geometry. A straightforward approach for determining the MS position is to solve the nonlinear equations [9] relating these measurements directly, but it is computationally intensive. Apart from the direct methodology, another common technique [10]-[12] that avoids solving the nonlinear equations is to linearize them, and then, the solution is found iteratively. However, this approach requires an initial estimate and cannot guarantee convergence to the correct solution unless the initial guess is close to it. To allow real-time implementation and ensure global optimization, we adopt the idea of the spherical interpolation (SI) in TDOA-based location [13] that reorganizes the nonlinear hyperbolic equations into a set of linear equations by introducing an intermediate variable, which is a function of the source position. However, the SI estimator solves the linear equations directly via least squares (LS) without using the known relation between the intermediate variable and the position coordinate. To improve the location accuracy of the SI approach, Chan and Ho have proposed [14] to use a two-stage weighted LS to solve for the source position by exploiting this relation implicitly, whereas [15] incorporates the relation explicitly by minimizing a constrained LS function based on the technique of Lagrange multipliers. According to [15], these two modified algorithms are referred to as the quadratic correction least squares (QCLS) and linear correction least squares (LCLS), respectively.

The rest of the paper is organized as follows. In Section II, the model for the TOA measurements is described. Two important performance measures of location accuracy, namely, the Cramér-Rao lower bound (CRLB) [16] and circular error probability (CEP) [12] are then reviewed. In Section III, we first derive a simple LS TOA-location algorithm via the introduction of a range parameter. An improved algorithm, which weighs the LS function and exploits the relation between the range variable and position coordinate, is then devised. Performance of the developed algorithms is analyzed in Section IV. Simulation results are presented in Section V to evaluate the location estimation performance of the two methods. Finally, conclusions are drawn in Section VI.

\section{TOA MeAsurement Model AND PeRformance MeAsures}

It is assumed that a reliable non-line-of-sight detection algorithm, such as [17] or [18], has first been employed to eliminate the measurements with large errors. As a result, all measurements we utilize for mobile location come from line-of-sight (LOS) propagation. Let $[x, y]$ be the MS position to be determined and the known coordinate of the $i$ th BS be $\left[x_{i}, y_{i}\right], i=1,2, \ldots, M$, where $M$ is the total number of receiving LOS BSs. The distance between the MS and the $i$ th BS, which is denoted by $d_{i}$, is given by

$$
d_{i}=\sqrt{\left(x-x_{i}\right)^{2}+\left(y-y_{i}\right)^{2}}, \quad i=1,2, \ldots, M .
$$


In the absence of measurement error, the one-way propagation time taken for the signal to travel from the MS to the $i$ th $\mathrm{BS}$, which is denoted by $t_{i}$, is

$$
t_{i}=\frac{d_{i}}{c}, \quad i=1,2, \ldots, M
$$

where $c$ is the speed of light. The range measurement based on $t_{i}$ in the presence of disturbance, which is denoted by $r_{i}$, is modeled as

$$
\begin{array}{r}
r_{i}=d_{i}+n_{i}=\sqrt{\left(x-x_{i}\right)^{2}+\left(y-y_{i}\right)^{2}}+n_{i} \\
i=1,2, \ldots, M
\end{array}
$$

where $n_{i}$ is the noise in $r_{i}$ or range error at the $i$ th BS. For ease of analysis, we assume that each measurement error $n_{i}$ is a zero-mean white Gaussian process with known variance $\sigma_{i}^{2}$. (The zero-mean assumption is valid as long as the multipath effect, if any, has been circumvented [19]. Although the parameters $\left\{\sigma_{i}^{2}\right\}$ are usually unknown in practice, they can be determined for a particular signaling type in the TOA-based location system by channel measurement. Once the variance estimates have been obtained, we consider them to be constants for all TOA measurements taken.)

The CRLB gives a lower bound on variance attainable by any unbiased estimators, and thus, it can be served as a benchmark to contrast with the mean square error of positioning algorithms. We show in Appendix A that the CRLBs for $x$ and $y$ are as in (4) and (5), shown at the bottom of the page. In addition to the CRLB, the CEP [12] is another approximate but simple performance measure of location accuracy. It is defined as the radius of the circle that has its center at the mean and contains half the realizations of the location estimates. If the location estimator is unbiased, the CEP is a measure of the uncertainty in the location estimate relative to the actual MS location. Therefore, the smaller the CEP, the more reliable the estimator should be. Note that an ellipse, which is characterized by its angle of rotation from the $\mathrm{x}$-axis, and the major and minor axes, can generally describe the contour that contains half the realizations of estimates better than the CEP circle. The complete procedures for computing this ellipse, as well as the CEP using the ML location estimate in Gaussian noise, can be found in [12]. Since the ML method should give optimum location estimates, the CEP using the ML location estimate is the optimal CEP.

\section{MobILE LOCATION AlgorithMS}

In this section, we develop two TOA-based mobile location algorithms using the SI principle. A simple LS mobile location estimator is first derived as follows. Without measurement errors, (3) becomes

$$
r_{i}=\sqrt{\left(x-x_{i}\right)^{2}+\left(y-y_{i}\right)^{2}}, \quad i=1,2, \ldots, M .
$$

Squaring both sides of (6) yields

$$
\begin{aligned}
r_{i}^{2} & =R^{2}-2 x x_{i}-2 y y_{i}+\left(x_{i}^{2}+y_{i}^{2}\right) \\
& \Rightarrow x_{i} x+y_{i} y-0.5 R^{2} \\
& =\frac{1}{2}\left(x_{i}^{2}+y_{i}^{2}-r_{i}^{2}\right), \quad i=1,2, \ldots, M
\end{aligned}
$$

where $R=\sqrt{x^{2}+y^{2}}$ is the range variable introduced in order to reorganize (6) into a set of linear equations in $x, y$ and $R^{2}$. Equation (7) can be expressed in matrix form as

$$
\mathbf{A} \theta=\mathbf{b}
$$

where

$$
\begin{aligned}
\mathbf{A} & =\left[\begin{array}{ccc}
x_{1} & y_{1} & -0.5 \\
\vdots & \vdots & \vdots \\
x_{M} & y_{M} & -0.5
\end{array}\right], \boldsymbol{\theta}=\left[\begin{array}{c}
x \\
y \\
R^{2}
\end{array}\right], \quad \text { and } \\
\mathbf{b} & =\frac{1}{2}\left[\begin{array}{c}
x_{1}^{2}+y_{1}^{2}-r_{1}^{2} \\
\vdots \\
x_{M}^{2}+y_{M}^{2}-r_{M}^{2}
\end{array}\right] .
\end{aligned}
$$

In the presence of measurement errors, $\boldsymbol{\theta}$ can be estimated using the standard LS

$$
\begin{aligned}
\hat{\boldsymbol{\theta}} & =\arg \min _{\breve{\boldsymbol{\theta}}}(\mathbf{A} \breve{\boldsymbol{\theta}}-\mathbf{b})^{T}(\mathbf{A} \breve{\boldsymbol{\theta}}-\mathbf{b}) \\
& =\left(\mathbf{A}^{T} \mathbf{A}\right)^{-1} \mathbf{A}^{T} \mathbf{b}
\end{aligned}
$$

where $\breve{\boldsymbol{\theta}}=\left[\breve{x}, \breve{y}, \breve{R}^{2}\right]^{T}$ represents an optimization variable vector.

For better performance, we can add a weighting matrix $\mathbf{W}$ to (9) and restrict $\boldsymbol{\theta}$ to satisfy the basic relationship

$$
\breve{R}=\sqrt{\breve{x}^{2}+\breve{y}^{2}}
$$

This leads to the following constrained optimization problem:

$$
\hat{\boldsymbol{\theta}}_{\mathrm{cW}}=\arg \min _{\breve{\boldsymbol{\theta}}}(\mathbf{A} \ddot{\boldsymbol{\theta}}-\mathbf{b})^{T} \mathbf{W}(\mathbf{A} \breve{\boldsymbol{\theta}}-\mathbf{b})
$$

subject to

$$
\mathbf{q}^{T} \breve{\boldsymbol{\theta}}+\breve{\boldsymbol{\theta}}^{T} \mathbf{P} \breve{\boldsymbol{\theta}}=0
$$

where

$$
\mathbf{P}=\left[\begin{array}{lll}
1 & 0 & 0 \\
0 & 1 & 0 \\
0 & 0 & 0
\end{array}\right] \text { and } \mathbf{q}=\left[\begin{array}{c}
0 \\
0 \\
-1
\end{array}\right]
$$

Here, (12) is a matrix characterization of the relation in (10).

Let us study the disturbance in $\mathbf{b}$, which will lead to a suggestion on the choice of $\mathbf{W}$ [14]. For sufficiently small measurement error or high signal-to-noise ratio (SNR) conditions, the squared value of $r_{i}$ can be approximated as

$$
r_{i}^{2}=\left(d_{i}+n_{i}\right)^{2} \approx d_{i}^{2}+2 d_{i} n_{i}, \quad i=1,2, \ldots, M .
$$

As a result, the disturbance between the true and measured squared distances is

$$
\varepsilon_{i}=r_{i}^{2}-d_{i}^{2} \approx 2 d_{i} n_{i}, \quad i=1,2, \ldots, M .
$$

In vector form, $\left\{\varepsilon_{i}\right\}$ are expressed as

$$
\varepsilon=\left[2 d_{1} n_{1}, 2 d_{2} n_{2}, \ldots, 2 d_{M} n_{M}\right]^{T} .
$$

The covariance matrix of the disturbance is thus of the form

$$
\Psi=E\left[\varepsilon \varepsilon^{T}\right]=\mathbf{B Q B}
$$

$$
\begin{aligned}
\operatorname{CRLB}(x) & =\frac{\sum_{i=1}^{M} \frac{\left(y-y_{i}\right)^{2}}{\sigma_{i}^{2}\left[\left(x-x_{i}\right)^{2}+\left(y-y_{i}\right)^{2}\right]}}{\left[\sum_{i=1}^{M} \frac{\left(x-x_{i}\right)^{2}}{\sigma_{i}^{2}\left[\left(x-x_{i}\right)^{2}+\left(y-y_{i}\right)^{2}\right]}\right]\left[\sum_{i=1}^{M} \frac{\left(y-y_{i}\right)^{2}}{\sigma_{i}^{2}\left[\left(x-x_{i}\right)^{2}+\left(y-y_{i}\right)^{2}\right]}\right]-\left[\sum_{i=1}^{M} \frac{\left(x-x_{i}\right)\left(y-y_{i}\right)}{\sigma_{i}^{2}\left[\left(x-x_{i}\right)^{2}+\left(y-y_{i}\right)^{2}\right]}\right]^{2}} \\
\operatorname{CRLB}(y) & =\frac{\sum_{i=1}^{M} \frac{\left(x-x_{i}\right)^{2}}{\sigma_{i}^{2}\left[\left(x-x_{i}\right)^{2}+\left(y-y_{i}\right)^{2}\right]}}{\left[\sum_{i=1}^{M} \frac{\left(x-x_{i}\right)^{2}}{\sigma_{i}^{2}\left[\left(x-x_{i}\right)^{2}+\left(y-y_{i}\right)^{2}\right]}\right]\left[\sum_{i=1}^{M} \frac{\left(y-y_{i}\right)^{2}}{\sigma_{i}^{2}\left[\left(x-x_{i}\right)^{2}+\left(y-y_{i}\right)^{2}\right]}\right]-\left[\sum_{i=1}^{M} \frac{\left(x-x_{i}\right)\left(y-y_{i}\right)}{\sigma_{i}^{2}\left[\left(x-x_{i}\right)^{2}+\left(y-y_{i}\right)^{2}\right]}\right]^{2}}
\end{aligned}
$$


where $E[\cdot]$ is the expectation operator, $\mathbf{B}=\operatorname{diag}\left(2 d_{1}\right.$, $\left.2 d_{2}, \ldots, 2 d_{M}\right)$, and $\mathbf{Q}=\operatorname{diag}\left(\sigma_{1}^{2}, \sigma_{2}^{2}, \ldots, \sigma_{M}^{2}\right)$. The optimum weighting matrix for (11) is given by $\mathbf{W}=\Psi^{-1}$. Since it depends on the unknown $\left\{d_{i}\right\}$, we use the approximate value of $\Psi \approx \hat{\mathbf{B}} \hat{\mathbf{B}}$, where $\hat{\mathbf{B}}=\operatorname{diag}\left(2 r_{1}, 2 r_{2}, \ldots, 2 r_{M}\right)$.

We now consider solving the constrained weighted least squares (CWLS) problem in (11) and (12), which is equivalent to minimizing the Lagrangian [15]

$$
\mathcal{L}(\breve{\boldsymbol{\theta}}, \lambda)=(\mathbf{A} \breve{\boldsymbol{\theta}}-\mathbf{b})^{T} \boldsymbol{\Psi}^{-1}(\mathbf{A} \breve{\boldsymbol{\theta}}-\mathbf{b})+\lambda\left(\mathbf{q}^{T} \breve{\boldsymbol{\theta}}+\breve{\boldsymbol{\theta}}^{T} \mathbf{P} \breve{\boldsymbol{\theta}}\right)
$$

where $\lambda$ is the Lagrange multiplier. We show in Appendix B that a minimum point for the CWLS problem, either global or local, is given by

$$
\hat{\boldsymbol{\theta}}_{\mathrm{cw}}=\left(\mathbf{A}^{T} \boldsymbol{\Psi}^{-1} \mathbf{A}+\lambda \mathbf{P}\right)^{-1}\left(\mathbf{A}^{T} \mathbf{\Psi}^{-1} \mathbf{b}-\frac{\lambda}{2} \mathbf{q}\right)
$$

where $\lambda$ is determined from the five-root equation

$$
\begin{aligned}
c_{3} f_{3} & -\frac{\lambda}{2} c_{3} g_{3}+\sum_{i=1}^{2} \frac{c_{i} f_{i}}{1+\lambda \gamma_{i}}-\frac{\lambda}{2} \sum_{i=1}^{2} \frac{c_{i} g_{i}}{1+\lambda \gamma_{i}} \\
+ & \sum_{i=1}^{2} \frac{e_{i} f_{i} \gamma_{i}}{\left(1+\lambda \gamma_{i}\right)^{2}}-\frac{\lambda}{2} \sum_{i=1}^{2} \frac{e_{i} g_{i} \gamma_{i}}{\left(1+\lambda \gamma_{i}\right)^{2}} \\
- & \frac{\lambda}{2} \sum_{i=1}^{2} \frac{c_{i} f_{i} \gamma_{i}}{\left(1+\lambda \gamma_{i}\right)^{2}}+\frac{\lambda^{2}}{4} \sum_{i=1}^{2} \frac{c_{i} g_{i} \gamma_{i}}{\left(1+\lambda \gamma_{i}\right)^{2}}=0
\end{aligned}
$$

and $\left\{c_{i}\right\},\left\{e_{i}\right\},\left\{f_{i}\right\}$, and $\left\{g_{i}\right\}, i=1,2,3$ have been defined in Appendix B. The desired $\lambda$ is found by the following procedure.

a) Obtain the five roots of (19) using a root-finding algorithm. Discard any complex roots because the Lagrange multiplier is always real for real optimization problems.

b) Put the real $\lambda$ 's back to (18), and obtain subestimates of $\hat{\boldsymbol{\theta}}_{\mathrm{cw}}$.

c) The subestimate that yields the smallest objective value of $(\mathbf{A} \boldsymbol{\theta}-$

b) ${ }^{T} \mathbf{W}(\mathbf{A} \breve{\boldsymbol{\theta}}-\mathbf{b})$ is taken as the globally optimal CWLS solution. Efficient numerical methods for root finding can be found in [20], and interested readers may refer to it. Note that we can follow the argument of [15] by finding the $\lambda$ whose value is closest to zero only in order to save computation.

\section{Performance Analysis}

In this section, the bias and variance of the proposed location algorithms under sufficiently high SNR conditions are analyzed. Based on (13), we define

$$
\overline{\mathbf{b}}=\frac{1}{2}\left[\begin{array}{c}
x_{1}^{2}+y_{1}^{2}-d_{1}^{2} \\
\vdots \\
x_{M}^{2}+y_{M}^{2}-d_{M}^{2}
\end{array}\right] \text { and } \quad \tilde{\mathbf{b}}=-\left[\begin{array}{c}
d_{1} n_{1} \\
\vdots \\
d_{M} n_{M}
\end{array}\right]
$$

The vectors $\overline{\mathbf{b}}$ and $\tilde{\mathbf{b}}$ are the noise-free and disturbance components of $\mathbf{b}$, respectively. The LS solution of (9) can then be written as

$$
\hat{\boldsymbol{\theta}}=\left(\mathbf{A}^{T} \mathbf{A}\right)^{-1} \mathbf{A}^{T}(\overline{\mathbf{b}}+\tilde{\mathbf{b}}) .
$$

Since $E[\tilde{\mathbf{b}}]$ is zero, taking the expected value of (20) yields $E[\hat{\boldsymbol{\theta}}]=$ $\left(\mathbf{A}^{T} \mathbf{A}\right)^{-1} \mathbf{A}^{T} \overline{\mathbf{b}}=\boldsymbol{\theta}$, which indicates the unbiasedness of the LS algorithm under sufficiently small noise conditions.

On the other hand, we follow [15] to derive the bias of the CWLS algorithm, which has the form

$$
\begin{aligned}
E\left[\hat{\boldsymbol{\theta}}_{\mathrm{cw}}\right] & -\boldsymbol{\theta} \\
= & -\frac{\lambda}{2}\left(\mathbf{A}^{T} \boldsymbol{\Psi}^{-1} \mathbf{A}\right)^{-1} \mathbf{q}+\sum_{n=1}^{\infty}\left(-\lambda\left(\mathbf{A}^{T} \boldsymbol{\Psi}^{-1} \mathbf{A}\right)^{-1} \mathbf{P}\right)^{n} \boldsymbol{\theta} \\
& -\frac{\lambda}{2} \sum_{n=1}^{\infty}\left(-\boldsymbol{\lambda}\left(\mathbf{A}^{T} \boldsymbol{\Psi}^{-1} \mathbf{A}\right)^{-1} \mathbf{P}\right)^{n}\left(\mathbf{A}^{T} \boldsymbol{\Psi}^{-1} \mathbf{A}\right)^{-1} \mathbf{q}
\end{aligned}
$$

for $\lambda<1 /\left\|\left(\mathbf{A}^{T} \mathbf{\Psi}^{-1} \mathbf{A}\right)^{-1} \mathbf{P}\right\|$. Although the CWLS estimator is biased, the bias magnitude will be very small as $\lambda$ should close to zero for sufficiently high SNR conditions, and this is also illustrated via simulation results in the following section. It is noteworthy that Huang et al. [15] have also demonstrated that the constrained algorithms, namely, the QLCS and LCLS methods, possess negligible biases.

Considering that $E\left[\hat{\boldsymbol{\theta}}_{\mathrm{cw}}\right] \approx \boldsymbol{\theta}$, the variances of the MS location estimate $\left[\hat{x}_{\mathrm{cw}}, \hat{y}_{\mathrm{cw}}\right]$ for the CWLS algorithm are derived as follows. We first notice that the solution for the constrained optimization problem in (11) and (12) is essentially

$$
\hat{\boldsymbol{\theta}}_{\mathrm{cw}}=\arg \min _{\{\breve{x}, \breve{y}\}} J_{\mathrm{cw}}
$$

where

$$
J_{\mathrm{cw}}=\sum_{i=1}^{M} w_{i}\left[x_{i} \breve{x}+y_{i} \breve{y}-0.5\left(\breve{x}^{2}+\breve{y}^{2}\right)-\frac{1}{2}\left(\breve{x}^{2}+\breve{y}^{2}-r_{i}^{2}\right)\right]^{2}
$$

because $\breve{R}^{2}$ should satisfy $\breve{R}^{2}=\breve{x}^{2}+\breve{y}^{2}$ according to (10). For simplicity, we consider $\mathbf{W}=\operatorname{diag}\left(w_{1}, w_{2}, \ldots, w_{M}\right)$, which agrees with our uncorrelated noise assumption. By extending a standard variance analysis technique [21], [22] to multiple parameter estimation, the variances of $\hat{x}_{\mathrm{cw}}$ and $\hat{y}_{\mathrm{cw}}$, when they are located in a reasonable proximity to $(x, y)$, are given by (see Appendix C) (24) and (25), shown at the bottom of the page. In Appendix C, we show that (24) and (25) are equivalent to the CRLB in (4) and (5), respectively, and this indicates that the CWLS algorithm is optimal under sufficiently high SNR conditions.

In a similar but more tedious manner, the variances of the MS location estimate $[\hat{x}, \hat{y}]$ for the LS algorithm have also been derived. Since their expressions are much more complicated and cannot give us any insightful findings, we omit them in this paper.

$$
\approx \frac{\operatorname{var}\left(\hat{x}_{\mathrm{cw}}\right)}{\left.\left\{E\left[\left(\frac{\partial J_{\mathrm{cw}}}{\partial \breve{x}}\right)^{2}\right]\left(E\left[\frac{\partial^{2} J_{\mathrm{cw}}}{\partial \breve{y}^{2}}\right]\right)^{2}-2 E\left[\frac{\partial^{2} J_{\mathrm{cw}}}{\partial \breve{x} \partial \breve{y}}\right] E\left[\frac{\partial^{2} J_{\mathrm{cw}}}{\partial \breve{y}^{2}}\right] E\left[\left(\frac{\partial J_{\mathrm{cw}}}{\partial \breve{x}}\right)\left(\frac{\partial J_{\mathrm{cw}}}{\partial \breve{y}}\right)\right]+E\left[\left(\frac{\partial J_{\mathrm{cw}}}{\partial \breve{y}}\right)^{2}\right]\left(E\left[\frac{\partial^{2} J_{\mathrm{cw}}}{\partial \breve{y}^{2}}\right]-\left(E\left[\frac{\partial^{2} J_{\mathrm{cw}}}{\partial \breve{x} \partial \breve{y}}\right]\right)^{2}\right\}^{2} J_{\mathrm{cw}}\right]\right)^{2} \mid} \mid
$$

$\operatorname{var}\left(\hat{y}_{\mathrm{cw}}\right)$

$$
\approx \frac{E\left[\left(\frac{\partial J_{\mathrm{cw}}}{\partial \breve{y}}\right)^{2}\right]\left(E\left[\frac{\partial^{2} J_{\mathrm{cw}}}{\partial \breve{x}^{2}}\right]\right)^{2}-2 E\left[\frac{\partial^{2} J_{\mathrm{cw}}}{\partial \breve{x} \partial \breve{y}}\right] E\left[\frac{\partial^{2} J_{\mathrm{cw}}}{\partial \breve{x}^{2}}\right] E\left[\left(\frac{\partial J_{\mathrm{cw}}}{\partial \breve{x}}\right)\left(\frac{\partial J_{\mathrm{cw}}}{\partial \breve{y}}\right)\right]+E\left[\left(\frac{\partial J_{\mathrm{cw}}}{\partial \breve{x}}\right)^{2}\right]\left(E\left[\frac{\partial^{2} J_{\mathrm{cw}}}{\partial \breve{x} \partial \ddot{y}}\right]\right)^{2} \mid}{\left\{E\left[\frac{\partial^{2} J_{\mathrm{cw}}}{\partial \breve{x}^{2}}\right] E\left[\frac{\partial^{2} J_{\mathrm{cw}}}{\partial \breve{y}^{2}}\right]-\left(E\left[\frac{\partial^{2} J_{\mathrm{cw}}}{\partial \breve{x} \partial \breve{y}}\right]\right)^{2}\right\}^{2}} \mid \begin{gathered}
x=x \\
\check{y}=y
\end{gathered}
$$




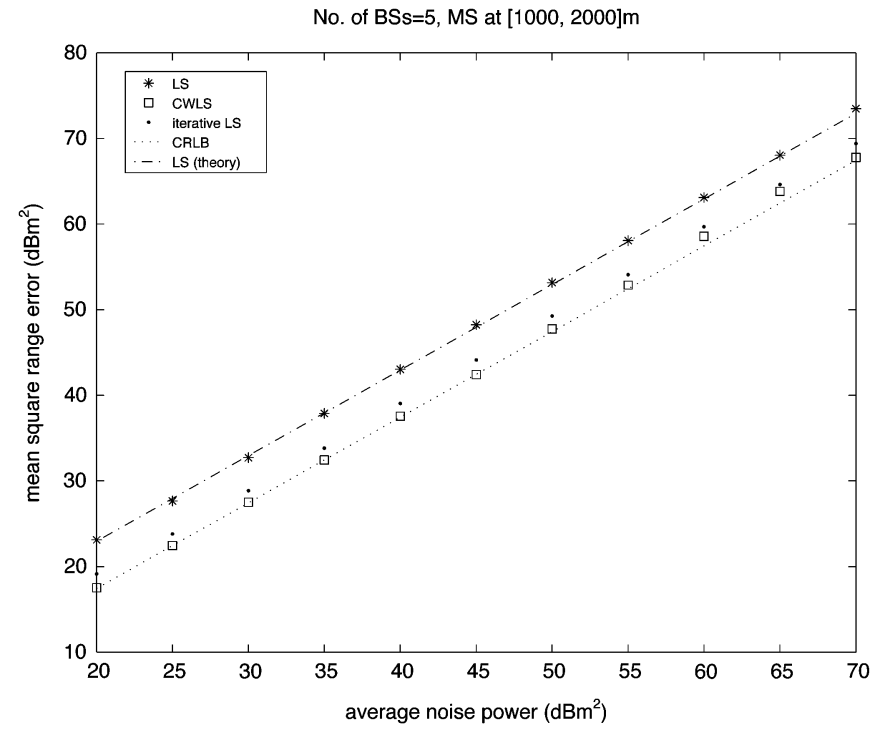

Fig. 1. Mean square range errors at $[x, y]=[1000,2000] \mathrm{m}$.

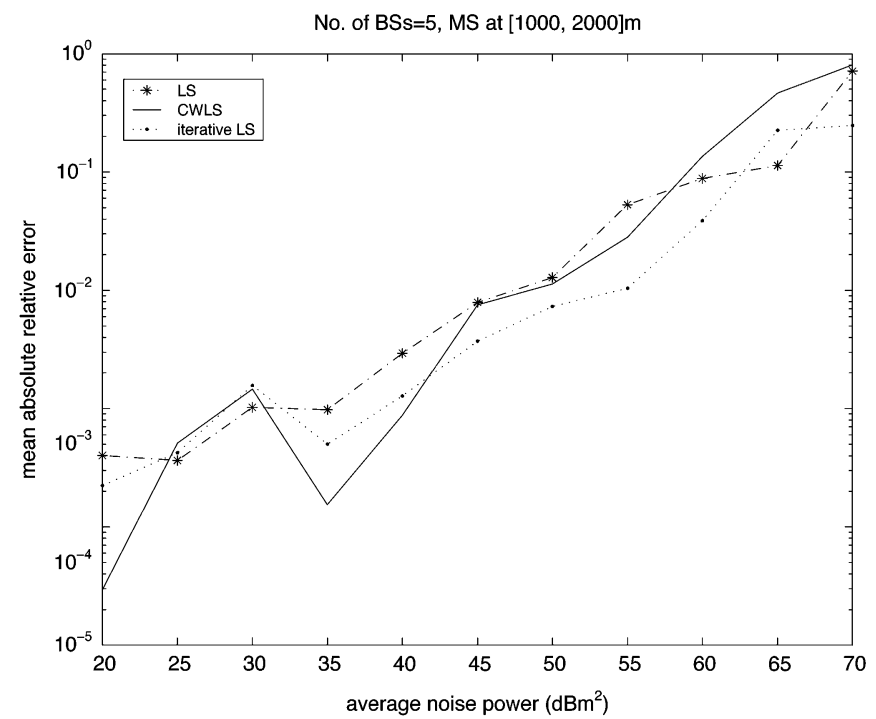

Fig. 2. Mean absolute relative errors at $[x, y]=[1000,2000] \mathrm{m}$.

\section{Simulation Results}

Computer simulations had been conducted to evaluate the performance of the proposed TOA-based location algorithms by comparing with the iterative LS technique [11] and CRLB. For [11], we used the actual MS position as the initial estimate in the iterative procedure to ensure global convergence. The CEPs of the LS and CWLS estimators were also studied and contrasted with those of the ML estimates. We considered a five-BS geometry with coordinates $[0,0] \mathrm{m}, \quad[3000 \sqrt{3}, 3000] \mathrm{m}, \quad[0,6000] \mathrm{m}, \quad[-3000 \sqrt{3}, 3000] \mathrm{m}$, and $[-3000 \sqrt{3},-3000] \mathrm{m}$. All results were averages of 1000 independent runs.

Fig. 1 plots the mean square range errors (MSREs) of the LS, CWLS, and iterative LS methods versus the average noise power at $[x, y]=$ $[1000,2000] \mathrm{m}$, where the noise refers to the range error. The theoretical range variance of the LS estimator at sufficiently high SNRs and that of the CWLS method or the CRLB were also included. The MSRE was defined as $E\left[(x-\hat{x})^{2}+(y-\hat{y})^{2}\right]$, and it has a linear relationship with the geometric dilution of precision (GDOP) [6], whereas the average noise power was given by $(1 / M) \sum_{i=1}^{M} \sigma_{i}^{2}$, where $\sigma_{i}^{2}$ was chosen such that all $\sigma_{i}^{2} / d_{i}^{2}$ were kept identical. It can be seen that the

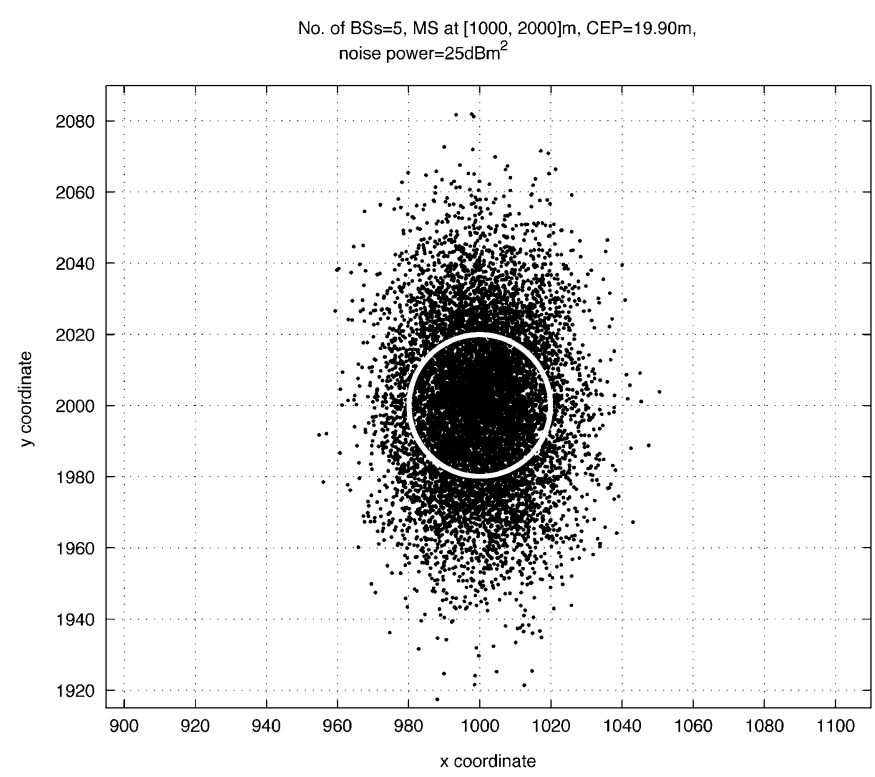

Fig. 3. MS estimate distribution of the LS estimator at $[x, y]=[1000,2000]$ $\mathrm{m}$ for average noise power of $25 \mathrm{~dB} \mathrm{\textrm {m } ^ { 2 }}$.

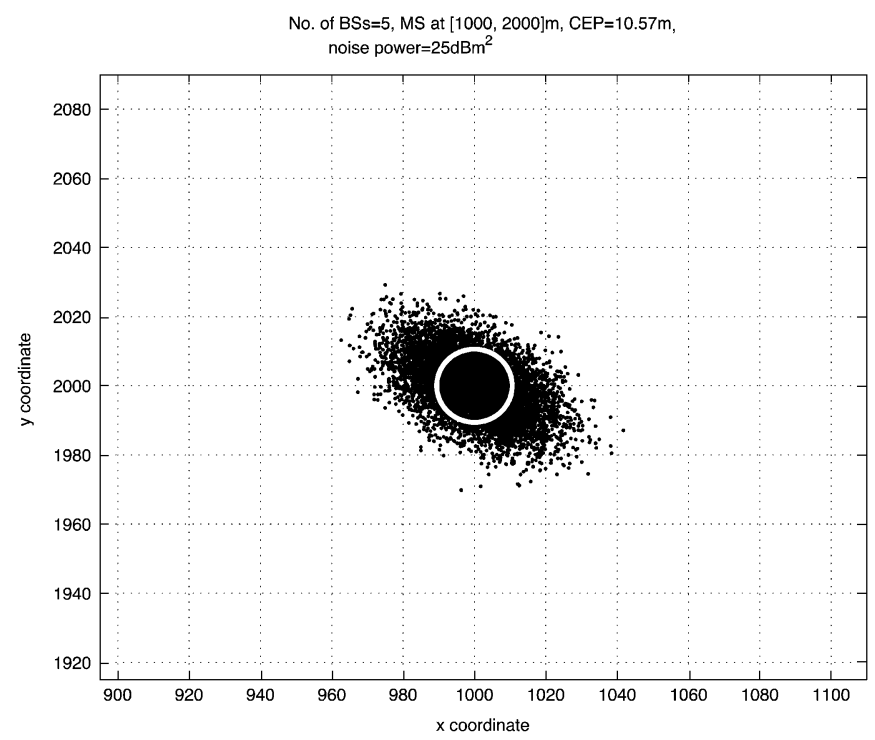

Fig. 4. MS estimate distribution of the CWLS estimator at $[x, y]=$ $[1000,2000] \mathrm{m}$ for average noise power of $25 \mathrm{~dB} \mathrm{~m}^{2}$.

performance of the CWLS method approached the CRLB, which verified its optimality as well as our analysis and outperformed the LS and iterative LS estimators by approximately 5 and $2 \mathrm{~dB} \mathrm{~m}^{2}$, respectively, for the whole range of noise powers. Moreover, there was good agreement between the MSRE and theoretical variance of the LS method. It is noteworthy that the average noise power range was reasonable for practical mobile location applications [23]. The mean absolute relative errors (MAREs) of the proposed methods and iterative LS estimator, which were defined as $|(E[\hat{x}]-x) / x|+|(E[\hat{y}]-y) / y|$, are shown in Fig. 2. We see that all methods had comparable MAREs, which implies that they had similar empirical biases. From Figs. 1 and 2, we know that these biases were in fact very small when comparing with their variances.

Figs. 3 and 4 show the distributions of the MS position estimates obtained by the LS and CWLS methods, respectively, at an average noise power of $25 \mathrm{~dB} \mathrm{~m}^{2}$. The circles in the figures were centered at the actual MS location of $[1000,2000] \mathrm{m}$ and included half of the location estimates. The radii or CEPs in Figs. 3 and 4 were found to 19.90 and 


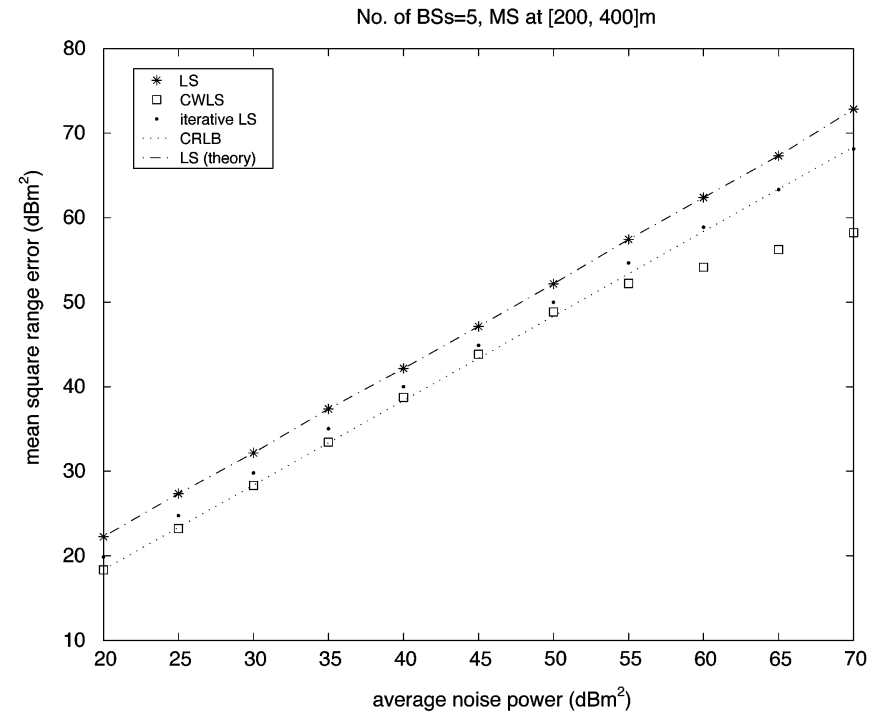

Fig. 5. Mean square range errors at $[x, y]=[200,400] \mathrm{m}$.

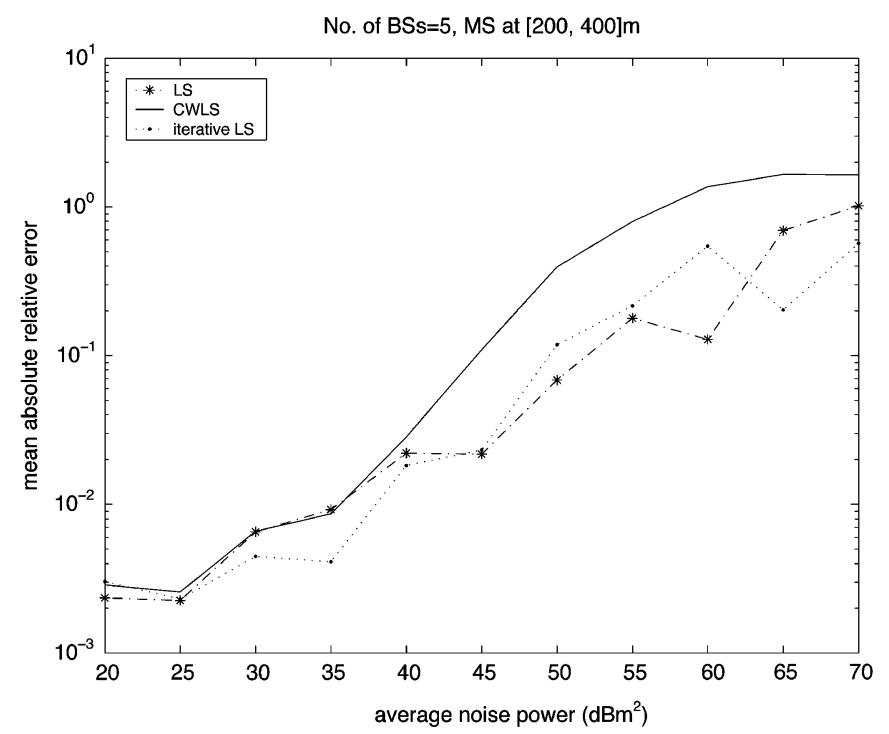

Fig. 6. Mean absolute relative errors at $[x, y]=[200,400] \mathrm{m}$.

$10.57 \mathrm{~m}$. This means that the CWLS estimator outperformed the LS method by approximately $9 \mathrm{~m}$ in terms of CEP. Furthermore, the CEP for the ML estimator was calculated as $10.45 \mathrm{~m}$, and thus, the optimality of the CWLS method is again demonstrated.

The above test was repeated for $[x, y]=[200,400] \mathrm{m}$, and the results are shown in Figs. 5 to 8. In Fig. 5, we see that the CWLS algorithm attained the CRLB and was superior to the LS and iterative LS methods by about 4 and $2 \mathrm{~dB} \mathrm{~m}^{2}$, respectively, when the average noise power was less than $55 \mathrm{~dB} \mathrm{~m}^{2}$. The performance of the CWLS method deviated from the CRLB for larger noise powers because the high SNR assumption for it broke down, although this assumption still held for the remaining methods. This finding also agreed with Fig. 6, where it is observed that the CWLS algorithm had larger MAREs than those of the LS and iterative LS estimators. In Fig. 7, the CEP of the LS method was found to be $18.64 \mathrm{~m}$, whereas in Fig. 8, we used an ellipse to include half of the location estimates because using a circle would introduce large errors. The semi-major and semi-minor axes of the ellipse were measured as 17.51 and $1.92 \mathrm{~m}$, respectively, which are close to those of the ellipse for ML estimation, which were computed as 17.26 and $1.76 \mathrm{~m}$.

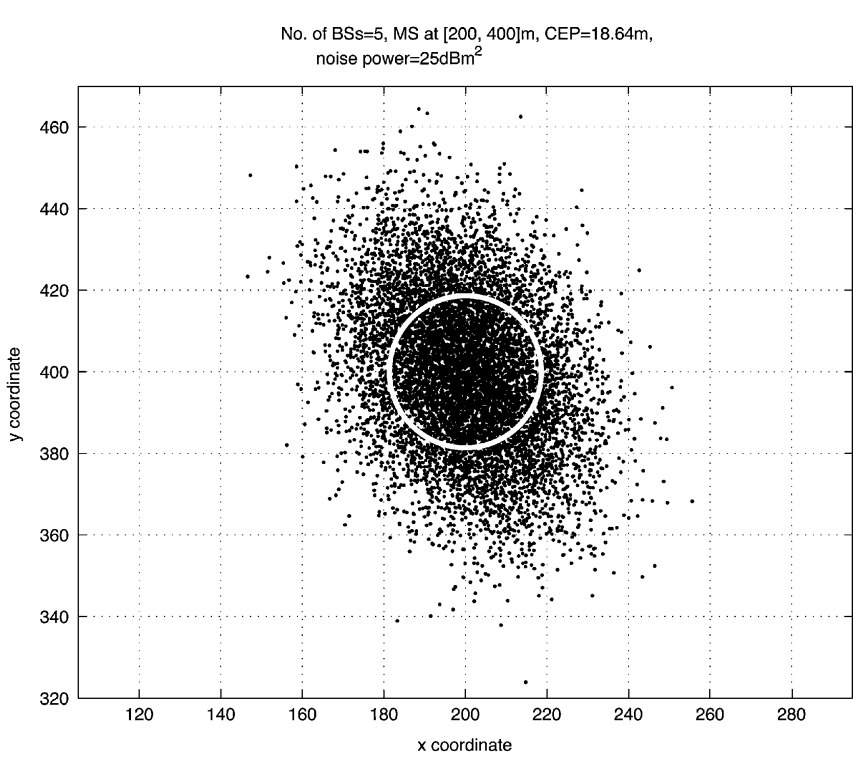

Fig. 7. MS estimate distribution of the LS estimator at $[x, y]=[200,400] \mathrm{m}$ for average noise power of $25 \mathrm{~dB} \mathrm{~m}^{2}$.

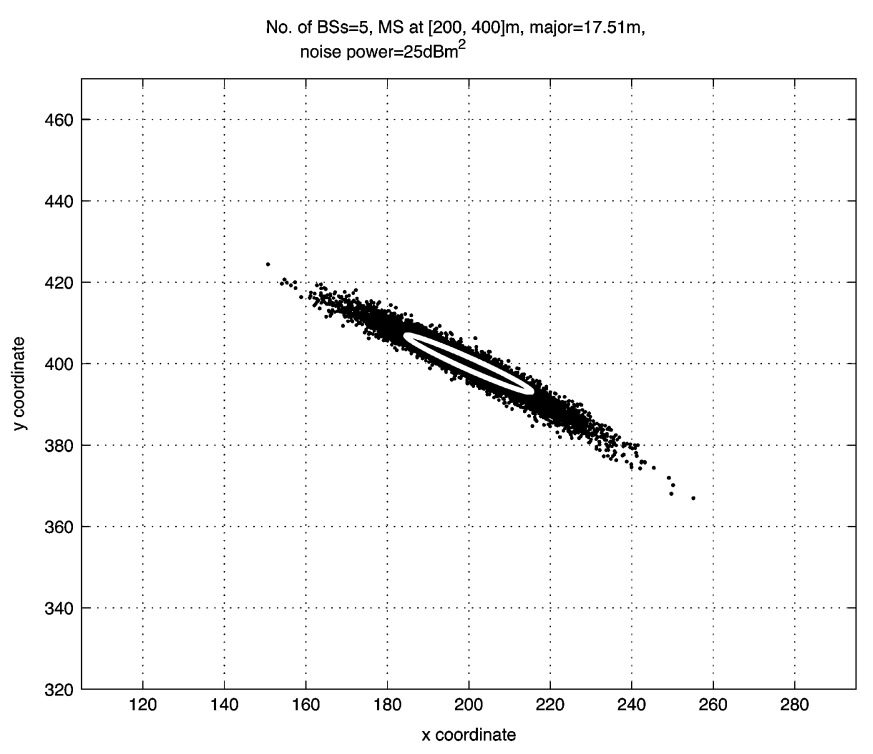

Fig. 8. MS estimate distribution of the CWLS estimator at $[x, y]=$ $[200,400] \mathrm{m}$ for average noise power of $25 \mathrm{~dB} \mathrm{~m}^{2}$.

\section{CONCLUSION}

Two time-of-arrival (TOA)-based location algorithms are developed from the spherical interpolation (SI) approach, which reorganizes nonlinear equations to linear equations via introduction of an intermediate variable. The first least squares algorithm directly extends the SI using the TOA measurements. The second constrained weighted least squares (CWLS) method is an improved version of the first algorithm with the use of weighting matrix and constraint. We have shown that the CWLS approach can attain the Cramér-Rao lower bound and optimal circular error probability under sufficiently small noise conditions.

\section{APPENDIX A}

Let $\mathbf{u}=\left[u_{1}, u_{2}\right]^{T}=[x, y]^{T}$. The CRLB for the $k$ th parameter of $\mathbf{u}, k=1,2$ is computed from [16]

$$
\operatorname{CRLB}\left(u_{k}\right)=\left[\mathbf{I}^{-1}(\mathbf{u})\right]_{k, k}, \quad k=1,2
$$


where

$$
[\mathbf{I}(\mathbf{u})]_{i, j}=-E\left[\frac{\partial^{2} \ln p(\mathbf{r} \mid \mathbf{u})}{\partial u_{i} \partial u_{j}}\right], \quad i=1,2, \quad \begin{aligned}
& \text { and } \\
& \quad j=1,2 \quad \text { (A. } 2)
\end{aligned}
$$

and

$$
\begin{aligned}
p(\mathbf{r} \mid \mathbf{u})= & \frac{1}{\sqrt{(2 \pi)^{M} \prod_{i=1}^{M} \sigma_{i}^{2}}} \\
& \cdot \exp \left\{\sum_{i=1}^{M} \frac{1}{2 \sigma_{i}^{2}}\left[r_{i}-\sqrt{\left(x-x_{i}\right)^{2}+\left(y-y_{i}\right)^{2}}\right]^{2}\right\}
\end{aligned}
$$

is the probability density function of $\mathbf{r}=\left[r_{1}, r_{2}, \ldots, r_{M}\right]^{T}$ conditioned on $\mathbf{u}$, and $[\mathbf{I}(\mathbf{u})]_{i, j}$ represents the $(i, j)$ th element of $\mathbf{I}(\mathbf{u})$, which is known as the Fisher information matrix and has the form of [8]

$\mathbf{I}(\mathbf{u})=\left[\begin{array}{lll}\sum_{i=1}^{M} \frac{\left(x-x_{i}\right)^{2}}{\sigma_{i}^{2}\left[\left(x-x_{i}\right)^{2}+\left(y-y_{i}\right)^{2}\right]} & \sum_{i=1}^{M} \frac{\left(x-x_{i}\right)\left(y-y_{i}\right)}{\sigma_{i}^{2}\left[\left(x-x_{i}\right)^{2}+\left(y-y_{i}\right)^{2}\right]} \\ \sum_{i=1}^{M} \frac{\left(x-x_{i}\right)\left(y-y_{i}\right)}{\sigma_{i}^{2}\left[\left(x-x_{i}\right)^{2}+\left(y-y_{i}\right)^{2}\right]} & \sum_{i=1}^{M} \frac{\left(y-y_{i}\right)^{2}}{\sigma_{i}^{2}\left[\left(x-x_{i}\right)^{2}+\left(y-y_{i}\right)^{2}\right]}\end{array}\right]$.

Taking the inverse of (A.4) yields the CRLBs for $x$ and $y$, which are given by (4) and (5).

\section{APPENDIX B}

The minimum of (17) is obtained by differentiating $\mathcal{L}(\breve{\boldsymbol{\theta}}, \lambda)$ with respect to $\breve{\boldsymbol{\theta}}$ and then equating the resultant expression to zero:

$$
\frac{\partial \mathcal{L}(\breve{\boldsymbol{\theta}}, \lambda)}{\partial \ddot{\boldsymbol{\theta}}}=2\left(\mathbf{A}^{T} \boldsymbol{\Psi}^{-1} \mathbf{A}+\lambda \mathbf{P}\right) \breve{\boldsymbol{\theta}}-2 \mathbf{A}^{T} \boldsymbol{\Psi}^{-1} \mathbf{b}+\lambda \mathbf{q}=0 .
$$

The solution to (A.5) is

$$
\hat{\boldsymbol{\theta}}_{\mathrm{cw}}=\left(\mathbf{A}^{T} \boldsymbol{\Psi}^{-1} \mathbf{A}+\lambda \mathbf{P}\right)^{-1}\left(\mathbf{A}^{T} \boldsymbol{\Psi}^{-1} \mathbf{b}-\frac{\lambda}{2} \mathbf{q}\right)
$$

where $\lambda$ has yet to be determined. To find $\lambda$, we substitute (A.6) into the equality constraint of (12):

$$
\begin{aligned}
\mathbf{q}^{T} & \left(\mathbf{A}^{T} \boldsymbol{\Psi}^{-1} \mathbf{A}+\lambda \mathbf{P}\right)^{-1}\left(\mathbf{A}^{T} \boldsymbol{\Psi}^{-1} \mathbf{b}-\frac{\lambda}{2} \mathbf{q}\right) \\
& +\left(\mathbf{b}^{T} \boldsymbol{\Psi}^{-1} \mathbf{A}-\frac{\lambda}{2} \mathbf{q}^{T}\right)\left(\mathbf{A}^{T} \boldsymbol{\Psi}^{-1} \mathbf{A}+\lambda \mathbf{P}\right)^{-1} \\
& \times \mathbf{P}\left(\mathbf{A}^{T} \boldsymbol{\Psi}^{-1} \mathbf{A}+\lambda \mathbf{P}\right)^{-1}\left(\mathbf{A}^{T} \boldsymbol{\Psi}^{-1} \mathbf{b}-\frac{\lambda}{2} \mathbf{q}\right)=0
\end{aligned}
$$

Note that the matrix $\left(\mathbf{A}^{T} \Psi^{-1} \mathbf{A}\right)^{-1} \mathbf{P}$ can be diagonalized as

$$
\left(\mathbf{A}^{T} \Psi^{-1} \mathbf{A}\right)^{-1} \mathbf{P}=\mathbf{U} \boldsymbol{\Lambda} \mathbf{U}^{-1}
$$

where $\boldsymbol{\Lambda}=\operatorname{diag}\left(\gamma_{1}, \gamma_{2}, \gamma_{3}\right)$ and $\gamma_{i}, i=1,2,3$ are the eigenvalues of the matrix $\left(\mathbf{A}^{T} \Psi^{-1} \mathbf{A}\right)^{-1} \mathbf{P}$. Substituting (A.8) into $\left(\mathbf{A}^{T} \mathbf{\Psi}^{-1} \mathbf{A}+\right.$ $\lambda \mathbf{P})^{-1}$ gives

$$
\left(\mathbf{A}^{T} \boldsymbol{\Psi}^{-1} \mathbf{A}+\lambda \mathbf{P}\right)^{-1}=\mathbf{U}(\mathbf{I}+\lambda \mathbf{\Lambda})^{-1} \mathbf{U}^{-1}\left(\mathbf{A}^{T} \boldsymbol{\Psi}^{-1} \mathbf{A}\right)^{-1} .
$$

Putting (A.9) into (A.7), we get

$$
\begin{aligned}
& \mathbf{c}^{T}(\mathbf{I}+\lambda \boldsymbol{\Lambda})^{-1} \mathbf{f}-\frac{\lambda}{2} \mathbf{c}^{T}(\mathbf{I}+\lambda \boldsymbol{\Lambda})^{-1} \mathbf{g} \\
& \quad+\mathbf{e}^{T}(\mathbf{I}+\lambda \boldsymbol{\Lambda})^{-1} \boldsymbol{\Lambda}(\mathbf{I}+\lambda \boldsymbol{\Lambda})^{-1} \mathbf{f} \\
& -\frac{\lambda}{2} \mathbf{e}^{T}(\mathbf{I}+\lambda \boldsymbol{\Lambda})^{-1} \boldsymbol{\Lambda}(\mathbf{I}+\lambda \boldsymbol{\Lambda})^{-1} \mathbf{g} \\
& \quad-\frac{\lambda}{2} \mathbf{c}^{T}(\mathbf{I}+\lambda \boldsymbol{\Lambda})^{-1} \boldsymbol{\Lambda}(\mathbf{I}+\lambda \boldsymbol{\Lambda})^{-1} \mathbf{f} \\
& \quad+\frac{\lambda^{2}}{4} \mathbf{c}^{T}(\mathbf{I}+\lambda \boldsymbol{\Lambda})^{-1} \boldsymbol{\Lambda}(\mathbf{I}+\lambda \boldsymbol{\Lambda})^{-1} \mathbf{g}=0
\end{aligned}
$$

where

$$
\begin{aligned}
\mathbf{c}^{T} & =\mathbf{q}^{T} \mathbf{U}=\left[c_{1}, c_{2}, c_{3}\right] \\
\mathbf{g} & =\mathbf{U}^{-1}\left(\mathbf{A}^{T} \boldsymbol{\Psi}^{-1} \mathbf{A}\right)^{-1} \mathbf{q}=\left[g_{1}, g_{2}, g_{3}\right]^{T} \\
\mathbf{e}^{T} & =\mathbf{b}^{T} \Psi^{-1} \mathbf{A} \mathbf{U}=\left[e_{1}, e_{2}, e_{3}\right] \\
\mathbf{f} & =\mathbf{U}^{-1}\left(\mathbf{A}^{T} \Psi^{-1} \mathbf{A}\right)^{-1} \mathbf{A} \Psi^{-1} \mathbf{b}=\left[f_{1}, f_{2}, f_{3}\right]^{T} .
\end{aligned}
$$

Since the matrix $\left(\mathbf{A}^{T} \Psi^{-1} \mathbf{A}\right)^{-1} \mathbf{P}$ is of rank 2, one of its eigenvalues, say, $\gamma_{3}$, must be zero. After expanding (A.10) and putting $\gamma_{3}=0$, (A.10) can be simplified to (19).

\section{APPENDIX C}

The variances of the MS location estimate for the CWLS algorithm are derived as follows. It is clear that $\left(\hat{x}_{\mathrm{cw}}, \hat{y}_{\mathrm{cw}}\right)$ satisfies

$$
\left.\frac{\partial J_{\mathrm{cw}}}{\partial \breve{x}}\right|_{\substack{\breve{x}=\hat{x}_{\mathrm{cw}} \\ \breve{y}=\hat{y}_{\mathrm{cw}}}}=\left.\frac{\partial J_{\mathrm{cw}}}{\partial \breve{y}}\right|_{\substack{\breve{x}=\hat{x}_{\mathrm{cw}} \\ \breve{y}=\hat{y}_{\mathrm{cw}}}}=0 .
$$

Let $f(\breve{x}, \breve{y})$ and $g(\breve{x}, \breve{y})$ be the derivatives of $J_{\mathrm{cw}}$ with respect to $\breve{x}$ or $\breve{y}$. When $\left(\hat{x}_{\mathrm{cw}}, \hat{y}_{\mathrm{cw}}\right)$ is located at a reasonable proximity of $(x, y)$, using Taylor's series to expand $f\left(\hat{x}_{\mathrm{cw}}, \hat{y}_{\mathrm{cw}}\right)$ around $(x, y)$ up to the first-order terms yields

$$
-f(x, y) \approx\left(\hat{x}_{\mathrm{cw}}-x\right) f_{x}(x, y)+\left(\hat{y}_{\mathrm{cw}}-y\right) f_{y}(x, y)
$$

where $f_{x}(x, y)$ and $f_{y}(x, y)$ denote the derivatives of $f(\breve{x}, \breve{y})$ with respect to $\breve{x}$ and $\breve{y}$ evaluated at $(x, y)$, respectively.

When the derivatives of $f(\breve{x}, \breve{y})$ are sufficiently smooth around the point $(x, y),(\mathrm{A} .12)$ can be approximated as [21], [22]

$-f(x, y) \approx\left(\hat{x}_{\mathrm{cw}}-x\right) E\left\{f_{x}(x, y)\right\}$

$$
+\left(\hat{y}_{\mathrm{cw}}-y\right) E\left\{f_{y}(x, y)\right\} .
$$

Similarly, we have

$-g(x, y) \approx\left(\hat{x}_{\mathrm{cw}}-x\right) E\left\{g_{x}(x, y)\right\}$

$$
+\left(\hat{y}_{\mathrm{cw}}-y\right) E\left\{g_{y}(x, y)\right\}
$$

where $g_{x}(x, y)$ and $g_{y}(x, y)$ are defined similarly. Multiplying (A.13) by (A.14) and then taking the expected value gives

$$
\begin{aligned}
E[ & f(x, y) g(x, y)] \\
& \approx E\left[\left(\hat{x}_{\mathrm{cw}}-x\right)^{2}\right] E\left[f_{x}(x, y)\right] E\left[g_{x}(x, y)\right] \\
& +E\left[\left(\hat{y}_{\mathrm{cw}}-y\right)^{2}\right] E\left[f_{y}(x, y)\right] E\left[g_{y}(x, y)\right] \\
& +E\left[\left(\hat{x}_{\mathrm{cw}}-x\right)\left(\hat{y}_{\mathrm{cw}}-y\right)\right]\left\{E\left[f_{x}(x, y)\right] E\left[g_{y}(x, y)\right]\right. \\
& \left.+E\left[f_{y}(x, y)\right] E\left[g_{x}(x, y)\right]\right\} .
\end{aligned}
$$

Considering that the CWLS algorithm is unbiased, the variances of $\hat{x}_{\mathrm{cw}}$ and $\hat{y}_{\mathrm{cw}} \operatorname{are} \operatorname{var}\left(\hat{x}_{\mathrm{cw}}\right)=E\left[\left(\hat{x}_{\mathrm{cw}}-x\right)^{2}\right]$ and $\operatorname{var}\left(\hat{y}_{\mathrm{cw}}\right)=E\left[\left(\hat{y}_{\mathrm{cw}}-y\right)^{2}\right]$, respectively, whereas their covariance is $\operatorname{cov}\left(\hat{x}_{\mathrm{cw}}, \hat{y}_{\mathrm{cw}}\right)=E\left[\left(\hat{x}_{\mathrm{cw}}-\right.\right.$

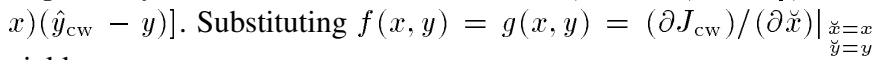
yields

$$
\begin{aligned}
\left.E\left[\left(\frac{\partial J_{\mathrm{cw}}}{\partial \breve{x}}\right)^{2}\right]\right|_{\substack{\breve{x}=x \\
\breve{y}=y}} & \left\{\operatorname{var}\left(\hat{x}_{\mathrm{cw}}\right)\left(E\left[\frac{\partial^{2} J_{\mathrm{cw}}}{\partial \breve{x}^{2}}\right]\right)^{2}\right. \\
& +\operatorname{var}\left(\hat{y}_{\mathrm{cw}}\right)\left(E\left[\frac{\partial^{2} J_{\mathrm{cw}}}{\partial \breve{x} \partial \breve{y}}\right]\right)^{2} \\
& \left.+2 \operatorname{cov}\left(\hat{x}_{\mathrm{cw}}, \hat{y}_{\mathrm{cw}}\right) E\left[\frac{\partial^{2} J_{\mathrm{cw}}}{\partial \breve{x}^{2}}\right] E\left[\frac{\partial^{2} J_{\mathrm{cw}}}{\partial \breve{x} \partial \breve{y}}\right]\right\}\left.\right|_{\substack{\breve{x}=x \\
\breve{y}=y}} .
\end{aligned}
$$


Putting $f(x, y)=g(x, y)=\left(\partial J_{\mathrm{cw}}\right) /\left.(\partial \breve{y})\right|_{\substack{x=x \\ y=y}}$, we get

$$
\begin{aligned}
\left.E\left[\left(\frac{\partial J_{\mathrm{cw}}}{\partial \breve{y}}\right)^{2}\right]\right|_{\substack{\breve{x}=x \\
\breve{y}=y}} & \left\{\operatorname{var}\left(\hat{x}_{\mathrm{cw}}\right)\left(E\left[\frac{\partial^{2} J_{\mathrm{cw}}}{\partial \breve{x} \partial \breve{y}}\right]\right)^{2}\right. \\
& +\operatorname{var}\left(\hat{y}_{\mathrm{cw}}\right)\left(E\left[\frac{\partial^{2} J_{\mathrm{cw}}}{\partial \breve{y}^{2}}\right]\right)^{2} \\
& \left.+2 \operatorname{cov}\left(\hat{x}_{\mathrm{cw}}, \hat{y}_{\mathrm{cw}}\right) E\left[\frac{\partial^{2} J_{\mathrm{cw}}}{\partial \breve{y}^{2}}\right] E\left[\frac{\partial^{2} J_{\mathrm{cw}}}{\partial \breve{x} \partial \breve{y}}\right]\right\}\left.\right|_{\substack{\breve{x}=x \\
\breve{y}=y}} .
\end{aligned}
$$

Furthermore, substituting $f(x, y)=\left(\partial J_{\mathrm{cw}}\right) /\left.(\partial \breve{x})\right|_{\substack{x=x \\ \breve{y}=y}}$ and $g(x, y)=$ $\left(\partial J_{\mathrm{cw}}\right) /\left.(\partial \breve{y})\right|_{\substack{\breve{x}=x \\ \breve{y}=y}}$ gives

$$
\begin{aligned}
\left.E\left[\left(\frac{\partial J_{\mathrm{cw}}}{\partial \breve{x}}\right)\left(\frac{\partial J_{\mathrm{cw}}}{\partial \breve{y}}\right)\right]\right|_{\substack{\breve{x}=x \\
\breve{y}=y}} & \left\{\operatorname{var}\left(\hat{x}_{\mathrm{cw}}\right) E\left[\frac{\partial^{2} J_{\mathrm{cw}}}{\partial \breve{x}^{2}}\right] E\left[\frac{\partial^{2} J_{\mathrm{cw}}}{\partial \breve{x} \partial \breve{y}}\right]\right. \\
& +\operatorname{var}\left(\hat{y}_{\mathrm{cw}}\right) E\left[\frac{\partial^{2} J_{\mathrm{cw}}}{\partial \breve{y}^{2}}\right] E\left[\frac{\partial^{2} J_{\mathrm{cw}}}{\partial \breve{x} \partial \breve{y}}\right] \\
& +\operatorname{cov}\left(\hat{x}_{\mathrm{cw}}, \hat{y}_{\mathrm{cw}}\right)\left\{E\left[\frac{\partial^{2} J_{\mathrm{cw}}}{\partial \breve{x}^{2}}\right] E\left[\frac{\partial^{2} J_{\mathrm{cw}}}{\partial \breve{y}^{2}}\right]\right. \\
& \left.\left.+\left(E\left[\frac{\partial^{2} J_{\mathrm{cw}}}{\partial \breve{x} \partial \breve{y}}\right]\right)^{2}\right\}\right\}\left.\right|_{\substack{\breve{x}=x \\
\breve{y}=y}} .
\end{aligned}
$$

Solving (A.16)-(A.18), we obtain (A.19) and (A.20), shown at the bottom of the page. The values of $E\left[\left(\left(\partial J_{\mathrm{cw}}\right) /(\partial \breve{x})\right)^{2}\right]$, $E\left[\left(\left(\partial J_{\mathrm{cw}}\right) /(\partial \breve{y})\right)^{2}\right], E\left[\left(\left(\partial J_{\mathrm{cw}}\right) /(\partial \breve{x})\right)\left(\left(\partial J_{\mathrm{cw}}\right) /(\partial \breve{y})\right)\right], E\left[\left(\partial^{2} J_{\mathrm{cw}}\right) /\right.$ $\left.(\partial \breve{x})^{2}\right], E\left[\left(\partial^{2} J_{\mathrm{cw}}\right) /(\partial \breve{y})^{2}\right]$, and $E\left[\left(\partial^{2} J_{\mathrm{cw}}\right) /(\partial \breve{x} \partial \breve{y})\right]$ at $(\breve{x}, \breve{y})=$ $(x, y)$ are now computed one by one.

Consider a sufficiently small noise condition such that the term $n_{i}^{2}$ can be ignored, and $w_{i} \approx\left(1 /\left(2 d_{i} \sigma_{i}\right)^{2}\right)$. Differentiating $J_{\mathrm{cw}}$ with respect to $\breve{x}$ gives

$$
\begin{aligned}
& \frac{\partial J_{\mathrm{cw}}}{\partial \breve{x}}=\sum_{i=1}^{M} 2 w_{i}\left[x_{i} \breve{x}+y_{i} \breve{y}-0.5\left(\breve{x}^{2}+\breve{y}^{2}\right)\right. \\
& \left.-\frac{1}{2}\left(\breve{x}^{2}+\breve{y}^{2}-r_{i}^{2}\right)\right]\left(x_{i}-\breve{x}\right) \\
& \left.\Rightarrow \frac{\partial J_{\mathrm{cw}}}{\partial \breve{x}}\right|_{\substack{x=x \\
\breve{y}=y}} \approx \sum_{i=1}^{M} 2 w_{i}\left[x_{i} x+y_{i} y-0.5\left(x^{2}+y^{2}\right)\right.
\end{aligned}
$$

$$
\begin{aligned}
& \left.\quad-\frac{1}{2}\left(x^{2}+y^{2}-d_{i}^{2}\right)\right]\left(x_{i}-x\right) \\
& +2 \sum_{i=1}^{M}\left(x_{i}-x\right) w_{i} d_{i} n_{i} \\
& \approx 2 \sum_{i=1}^{M} \frac{\left(x_{i}-x\right) d_{i} n_{i}}{\left(2 d_{i} \sigma_{i}\right)^{2}}
\end{aligned}
$$

Squaring (A.21) and taking the expected value, we obtain

$$
\left.E\left[\left(\frac{\partial J_{\mathrm{cw}}}{\partial \breve{x}}\right)^{2}\right]\right|_{\substack{\breve{x}=x \\ \breve{y}=y}} \approx \sum_{i=1}^{M} \frac{\left(x-x_{i}\right)^{2}}{4 \sigma_{i}^{2}\left[\left(x-x_{i}\right)^{2}+\left(y-y_{i}\right)^{2}\right]} .
$$

Similarly, $\left.E\left[\left(\left(\partial J_{\mathrm{cw}}\right) /(\partial \breve{y})\right)^{2}\right]\right|_{\substack{\breve{y}=x \\ \breve{y}=y}}$ and $E\left[\left(\left(\partial J_{\mathrm{cw}}\right) /(\partial \breve{x})\right)\left(\left(\partial J_{\mathrm{cw}}\right) /\right.\right.$

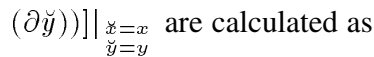

$$
\begin{gathered}
\left.E\left[\left(\frac{\partial J_{\mathrm{cw}}}{\partial \breve{y}}\right)^{2}\right]\right|_{\substack{\breve{s}=x \\
\breve{y}=y}} \\
\approx \sum_{i=1}^{M} \frac{\left(y-y_{i}\right)^{2}}{4 \sigma_{i}^{2}\left[\left(x-x_{i}\right)^{2}+\left(y-y_{i}\right)^{2}\right]}
\end{gathered}
$$

and

$$
\begin{gathered}
\left.E\left[\left(\frac{\partial J_{\mathrm{cw}}}{\partial \breve{x}}\right)\left(\frac{\partial J_{\mathrm{cw}}}{\partial \breve{y}}\right)\right]\right|_{\substack{x=x \\
\grave{y}=y}} \\
\approx \sum_{i=1}^{M} \frac{\left(x_{i}-x\right)\left(y_{i}-y\right)}{4 \sigma_{i}^{2}\left[\left(x-x_{i}\right)^{2}+\left(y-y_{i}\right)^{2}\right]} .
\end{gathered}
$$

Based on (A.21), we have

$$
\left.\frac{\partial^{2} J_{\mathrm{cw}}}{\partial \breve{x}^{2}}\right|_{\substack{\breve{x}=x \\ \breve{y}=y}} \approx \sum_{i=1}^{M} \frac{2\left(x-x_{i}\right)^{2}}{\left(2 d_{i} \sigma_{i}\right)^{2}}-\sum_{i=1}^{M} \frac{2 d_{i n_{i}}}{\left(2 d_{i} \sigma_{i}\right)^{2}} .
$$

Taking expected value of (A.25) yields

$$
\left.E\left[\frac{\partial^{2} J_{\mathrm{cw}}}{\partial \breve{x}^{2}}\right]\right|_{\substack{x=x \\ y=y}} \approx \sum_{i=1}^{M} \frac{\left(x-x_{i}\right)^{2}}{2 \sigma_{i}^{2}\left[\left(x-x_{i}\right)^{2}+\left(y-y_{i}\right)^{2}\right]} .
$$

In a similar manner, $\left.E\left[\left(\partial^{2} J_{\mathrm{cw}}\right) /(\partial \breve{y})^{2}\right]\right|_{\substack{\breve{y}=x \\ \breve{y}=y}}$ and $E\left[\left(\partial^{2} J_{\mathrm{cw}}\right) /\right.$ $(\partial \breve{x} \partial \breve{y})]\left.\right|_{\substack{\breve{x}=x \\ \breve{y}=y}}$ are calculated as

$$
\left.E\left[\frac{\partial^{2} J_{\mathrm{cw}}}{\partial \breve{y}^{2}}\right]\right|_{\substack{x=x \\ \breve{y}=y}} \approx \sum_{i=1}^{M} \frac{\left(y-y_{i}\right)^{2}}{2 \sigma_{i}^{2}\left[\left(x-x_{i}\right)^{2}+\left(y-y_{i}\right)^{2}\right]}
$$

$\operatorname{var}\left(\hat{x}_{\mathrm{cW}}\right)$

and

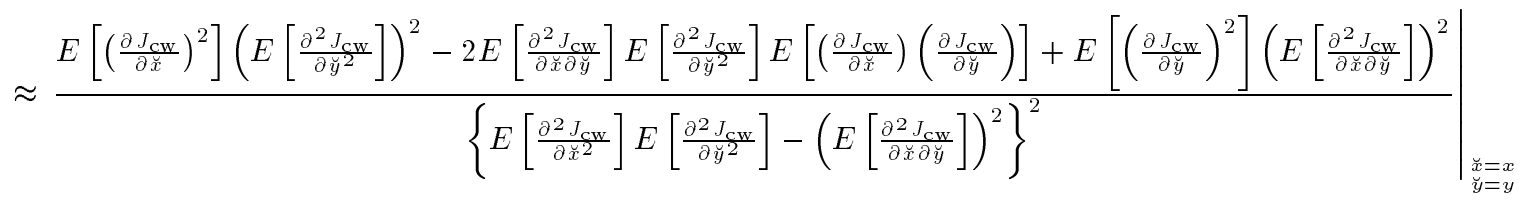

$\operatorname{var}\left(\hat{y}_{\mathrm{cw}}\right)$

$$
\approx \frac{E\left[\left(\frac{\partial J_{\mathrm{cw}}}{\partial \breve{y}}\right)^{2}\right]\left(E\left[\frac{\partial^{2} J_{\mathrm{cw}}}{\partial \breve{x}^{2}}\right]\right)^{2}-2 E\left[\frac{\partial^{2} J_{\mathrm{cw}}}{\partial \breve{x} \partial \breve{y}}\right] E\left[\frac{\partial^{2} J_{\mathrm{cw}}}{\partial \breve{x}^{2}}\right] E\left[\left(\frac{\partial J_{\mathrm{cw}}}{\partial \breve{x}}\right)\left(\frac{\partial J_{\mathrm{cw}}}{\partial \breve{y}}\right)\right]+E\left[\left(\frac{\partial J_{\mathrm{cw}}}{\partial \breve{x}}\right)^{2}\right]\left(E\left[\frac{\partial^{2} J_{\mathrm{cw}}}{\partial \breve{x} \breve{y}}\right]\right)^{2} \mid}{\left\{E\left[\frac{\partial^{2} J_{\mathrm{cw}}}{\partial \breve{x}^{2}}\right] E\left[\frac{\partial^{2} J_{\mathrm{cw}}}{\partial \breve{y}^{2}}\right]-\left(E\left[\frac{\partial^{2} J_{\mathrm{cW}}}{\partial \breve{x} \partial \breve{y}}\right]\right)^{2}\right\}^{2}} \cdot
$$


and

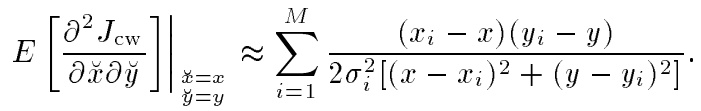

Substituting (A.22)-(A.24) and (A.26)-(A.28) into (A.19) and (A.20), it can be easily shown that they are equivalent to (4) and (5).

\section{ACKNOWLEDGMENT}

The authors would like to thank the anonymous reviewers for their careful reading and constructive suggestions that improved the quality of this correspondence.

\section{REFERENCES}

[1] Federal Commun. Commission [Online]. Available: http://www.fcc. gov/e911

[2] C. Drane, M. Macnaughtan, and C. Scott, "Positioning GSM telephones," IEEE Commun. Mag., pp. 46-54, Apr. 1998.

[3] H. Koshima and J. Hosen, "Personal locator services emerge," IEEE Spectrum, pp. 41-48, Feb. 2000.

[4] Y. Zhao, "Mobile phone location determination and its impact on intelligent transport systems," IEEE Trans. Intell. Transport. Syst., vol. 1, pp. 55-64, Mar. 2000

[5] D. Porcino, "Performance of a OTDOA-IPDL positioning receiver for 3G-FDD mode," in Proc. Int. Conf. 3G Mobile Commun. Technol., 2001, pp. $221-225$.

[6] J. J. Caffery Jr., Wireless Location in CDMA Cellular Radio Systems. Boston, MA: Kluwer, 2000.

[7] J. C. Liberti and T. S. Rappaport, Smart Antennas for Wireless Communications: IS-95 and Third Generation CDMA Applications. Upper Saddle River, NJ: Prentice-Hall, 1999.

[8] M. McGuire and K. N. Plataniotis, "A comparison of radiolocation for mobile terminals by distance measurements," in Proc. Int. Conf. Wireless Commun., 2000, pp. 1356-1359.

[9] J. Caffery and G. L. Stuber, "Subscriber location in CDMA cellular networks," IEEE Trans. Veh. Technol., vol. 47, pp. 406-416, May 1998.

[10] M. A. Spirito, "On the accuracy of cellular mobile station location estimation," IEEE Trans. Veh. Technol., vol. 50, pp. 674-685, May 2001

[11] W. H. Foy, "Position-location solutions by Taylor-series estimation," IEEE Trans. Aerosp. Electron. Syst., vol. AES-12, pp. 187-194, Mar 1976.

[12] D. J. Torrieri, "Statistical theory of passive location systems," IEEE Trans. Aerosp. Electron. Syst., vol. AES-20, pp. 183-197, Mar. 1984.

[13] J. O. Smith and J. S. Abel, "Closed-form least-squares source location estimation from range-difference measurements," IEEE Trans. Acoust. Speech, Signal Processing, vol. ASSP-35, pp. 1661-1669, Dec. 1987.

[14] Y. T. Chan and K. C. Ho, "A simple and efficient estimator for hyperbolic location," IEEE Trans. Signal Processing, vol. 42, pp. 1905-1915, Aug. 1994.

[15] Y. Huang, J. Benesty, G. W. Elko, and R. M. Mersereau, "Real-time passive source localization: A practical linear-correction least-squares approach," IEEE Trans. Speech, Audio Processing, vol. 9, pp. 943-956, Nov. 2001.

[16] S. M. Kay, Fundamentals of Statistical Signal Processing: Estimation Theory. Englewood Cliffs, NJ: Prentice-Hall, 1993, p. 44.

[17] P. C. Chen, "A nonline-of-sight error mitigation algorithm in location estimation," in Proc. IEEE Wireless Commun. Networking Conf., vol. 1, New Orleans, LA, 1999, pp. 316-320.

[18] S. Al-Jazzar, J. Caffery Jr., and H. R. You, "A scattering model based approach to NLOS mitigation in TOA location systems," in Proc. IEEE 55th Veh. Technol. Conf., vol. 2, Spring 2002, pp. 861-865.

[19] J. J. Fuchs, "Multipath time-delay detection and estimation," IEEE Trans. Signal Processing, vol. 47, pp. 237-243, Jan. 1999.

[20] W. H. Press, Numerical Recipes in C/C++. Cambridge, U.K.: Cambridge Univ. Press, 2002

[21] V. H. MacDonald and P. M. Schultheiss, "Optimum passive bearing estimation in a spatially incoherent noise environment," J. Acoust. Soc. Amer., vol. 46, no. 1, pp. 37-43, 1969.

[22] J. P. Ianniello, "Large and small error performance limits for multipath time delay estimation," IEEE Trans. Acoust., Speech, Signal Processing, vol. ASSP-34, pp. 245-251, Apr. 1986

[23] C. Andersson, GPRS and 3G Wireless Applications: Professional De veloper's Guide. New York: Wiley, 2001, p. 260.

\section{Reformulation of Pisarenko Harmonic Decomposition Method for Single-Tone Frequency Estimation}

\author{
H. C. So and K. W. Chan
}

\begin{abstract}
Based on the linear prediction (LP) property of sinusoidal signals, a closed-form unbiased frequency estimator for a real sinusoid in white noise is proposed. The frequency estimator, which is derived by minimizing a constrained least squares cost function, can be considered as a reformulation of the well known Pisarenko harmonic decomposer (PHD). Online computation of the frequency estimate can be achieved in a very simple manner, and its variance is derived. Computer simulations are included to corroborate the theoretical development and to contrast the estimator performance with the PHD, maximum likelihood, and LP-based methods as well as Cramér-Rao lower bound.
\end{abstract}

Index Terms-Constrained optimization, frequency estimation, low complexity, online algorithm, Pisarenko's method, real sinusoid.

\section{INTRODUCTION}

Frequency estimation of sinusoidal signals in noise is a frequently addressed problem in the signal processing literature, and it has a wide variety of applications such as angle-of-arrival estimation, demodulation of frequency-shift keying (FSK) signals, speech analysis, Doppler rate estimation, and measurements [1]-[4].

For a complex sinusoid in white noise, it is well known that the maximum likelihood (ML) estimate of frequency is obtained from the periodogram maximum [1]. Kenefic and Nuttall [5] have extended the problem to ML frequency estimation of a real tone and the optimum estimator maximizes a highly nonlinear and multimodal cost function. For both cases, the ML methods involve extensive computations, and this will be prohibitive in applications where rapid frequency estimation is required. Apart from the ML estimators, other frequency estimation techniques [3] include notch filtering, Capon methods, linear prediction (LP), Yule-Walker methods, and subspace-based approaches. Among the subspace-based methods, the Pisarenko harmonic decomposer (PHD) [6] is of historical interest because it was the first to exploit the eigenstructure of the covariance matrix, and its performance has been extensively studied [7]-[12]. Interestingly, the PHD for a single real sinusoid can be implemented in a very simple way [10], [11].

In this correspondence, we will focus on fast algorithm for frequency estimation of a real-valued tone in white noise. Our major contributions include

1) development of a computationally simple and unbiased single real tone frequency estimator. Although the proposed algorithm is similar to the PHD [10], [11], it generally has higher frequency estimation accuracy;

2) derivation of the small-sample variance of the frequency estimate, which means that the variance formula applies even for a small number of measurements.

The rest of the paper is organized as follows. In Section II, it is shown that the least squares (LS) cost function derived from the LP approach for a real-valued tone should be minimized subject to con-

Manuscript received February 25, 2003; revised May 30, 2003. The associate editor coordinating the review of this manuscript and approving it for publication was Dr. Markus Rupp.

The authors are with the Department of Computer Engineering and Information Technology, City University of Hong Kong, Kowloon, Hong Kong (e-mail ithcso@cityu.edu.hk; k.w.chan@student.cityu.edu.hk)

Digital Object Identifier 10.1109/TSP.2004.823473 